Meta

Journal des traducteurs

Translators' Journal

\title{
Niveaux linguistiques et terminologie de l'intelligence artificielle
}

\section{Silvia Pavel}

Volume 34, numéro 3, septembre 1989

1. Actes du Colloque Les terminologies spécialisées: Approches quantitative et logico-sémantique et 2 . Actes du Colloque Terminologie et Industries de la langue

URI : https://id.erudit.org/iderudit/003807ar

DOI : https://doi.org/10.7202/003807ar

Aller au sommaire du numéro

Éditeur(s)

Les Presses de l'Université de Montréal

ISSN

0026-0452 (imprimé)

1492-1421 (numérique)

Découvrir la revue

Citer cet article

Pavel, S. (1989). Niveaux linguistiques et terminologie de l'intelligence artificielle. Meta, 34(3), 344-351. https://doi.org/10.7202/003807ar d'utilisation que vous pouvez consulter en ligne. 


\title{
NIVEAUX LINGUISTIQUES ET TERMINOLOGIE DE L'INTELLIGENCE ARTIFICIELLE
}

\author{
SYlVIa PAVEL \\ Direction de la terminologie, Secrétariat d'État \\ Ottawa, Ontario, Canada
}

\begin{abstract}
Mon exposé aborde la problématique des terminologies naissantes en spécialités de pointe sous l'angle de leurs multiples réalisations dans un continuum intertextuel. En décrivant la communauté de l'IA, son champ d'investigation et la variété de ses discours, je compte apporter des éléments de preuve aux hypothèses suivantes : primo, la dynamique lexicale des différents types de discours technoscientifique est distincte et inséparable de la dynamique de leur production - l'analyse rhétorique, syntaxique et stylistique de ces discours ne peut ignorer les mécanismes de renouvellement lexical et réciproquement; secundo, l'analyse terminologique de l'intertexte technoscientifique en fonction de ses variables socio-culturelles permet de poser des distinctions réelles entre les niveaux linguistiques et d'en souligner les relations; tertio, les techniques IA en matière d'extraction de l'expertise par le cogniticien et de traitement symbolique du langage naturel présentent un intérêt certain pour la recherche terminologique dans les spécialités de pointe.
\end{abstract}

Trente ans après l'apparition de l'«intelligence artificielle», la polémique autour de son statut continue sans relâche. L'automne dernier, la prestigieuse revue française Le Débat la saluait comme «nouvelle science de l'esprit» tandis que Languages la reléguait aux technologies et disciplines médiatrices.

Pour ceux qui s'y réclament, l'IA est une science, non pas selon la définition classique de «connaissance structurée autour d'une théorie vérifiable par une méthode d'investigation donnée» - ce qui exige du chercheur une foi tenace dans la justesse des théories supportées par les faits - mais une science au sens donné en 1973 par Imre Lakatos «programme de recherches structuré autour d'une hypothèse, étayé d'un solide système de résolution de problèmes et capable de prédire des faits nouveaux, insoupçonnés et même contredits auparavant». Cette conception moderne de la scientificité n'exclut pas le scepticisme du chercheur quant à la validité d'une hypothèse, les critiques de $\mathrm{H}$. Dreyfus vis-à-vis l'IA analytique l'illustrent autant que les doutes de Jacques Arsac (1987) quant à la formalisation du sens. Mais elle n'exclut non plus le pari sur l'avenir des pionniers de l'IA tels McCarthy, Barr, Newell, Simon, Minsky, Papert ou Feigenbaum.

Les fondements théoriques de l'IA se placent au confluent des neurosciences, de la psychologie cognitive, de la linguistique, de la philosophie, de la logique et de l'informatique. Elle s'approprie et reformule les concepts de ces disciplines pour étudier l'ensemble des phénomènes constitutifs de l'intelligence humaine. Elle vérifie ses modèles et théories des processus cognitifs non pas sur des sujets humains mais sur des programmes informatiques. En prenant l'ordinateur comme outil et objet de ses recherches, l'IA se donne pour but, la construction de mécanismes présentant les caractéristiques d'un comportement intelligent. Elle développe toute une technologie de la 
connaissance programmable en systèmes logiciels sans perdre de vue son objectif à long terme: simuler la non-programmabilité du cerveau humain. C'est par le biais des systèmes experts que l'IA se rattache au génie logiciel et qu'elle se voit parfois réduite à une ingénierie ou une technique informatique.

Le terme «intelligence artificielle» est tout aussi loin de faire l'unanimité que le statut de la spécialité qu'il désigne : intelligence-machine, intelligence informatique et intelligence logicielle lui revendiquent des territoires autant que science cognitive (au singulier), intellectique, intellectronique, cognitique, ingénierie de la connaissance et génie cognitif. Chaque nouvelle dénomination est tour à tour définie, défendue, redéfinie, ridiculisée, reprise ici et rejetée là.

Pour le terminologue à la recherche d'un intertexte spécifique de 1'IA, analysable en termes de notions, de dénominations et d'usages, l'intérêt de ces discussions réside au delà du constat de technophobie (Hottois : 1988) et de variation technolectale - dans la mise en évidence des relations étroites entre les volets théorique et pratique de l'IA, et dans la délimitation sans cesse reprise du champ d'investigation d'une spécialité qui se constate mieux qu'elle ne se définit.

De cette constante remise en question surgit le portrait d'une communauté intellectuelle composée d'universitaires, de chercheurs, d'entrepreneurs et d'ingénieurs maîtrisant les moyens de s'exprimer, de s'affirmer et de persuader, et déterminée à innover au delà des savoirs acquis. Une communauté enfin, dont la cohésion interne semble augmenter avec l'intensité des polémiques et la rapidité des échanges d'idées. D'ailleurs, cette force cohésive des échanges ne se reflète qu'en partie dans les publications, les colloques, les téléconférences et les salons IA ou dans les importants projets de recherche où collaborent des associations professionnelles, des instituts et des laboratoires de plusieurs pays. En partie seulement, car l'économie des échanges linguistiques non standard - par ordinateur, par téléphone, par correspondance ou face-à-face est encore peu étudiée en français.

La langue de ces spécialistes du savoir et de l'esprit relève donc d'une multitude de «discours-en-mouvement» qui invoquent constamment un ensemble flou de notions récurrentes mais évolutives et de dénominations soumises aux réajustements de maintes reformulations. Le discours du spécialiste IA n'est pas le même dans un colloque ou au laboratoire. Il diffère de celui tenu devant des collègues d'une discipline distincte. Le language du professeur devant ses étudiants diffère de celui qu'il tient aux lecteurs de revues de vulgarisation ou aux téléspectateurs d'émissions technoscientifiques. D'autre part, le rédacteur technique ou le traducteur d'articles et de manuels sur l'IA n'étant pas forcément un spécialiste du domaine, vise la proximité plus que l'identité du discours spécialisé.

Décrire l'usage et l'efficacité de la communication à l'intérieur et entre ces divers groupes de spécialistes implique la prise en compte de variables socioculturelles telles l'intention de l'énonciateur, les connaissances du destinataire, la charge intellectuelle et affective du message et les ressources situationnelles (attentes et ententes tacites des interlocuteurs).

En fonction de ces variables, l'enseignant d'une langue de spécialité peut dégager les particularités syntaxiques et stylistiques de chaque type de discours - longueur des phrases, structures non personnelles, complémentations nominales, classes d'énoncés définitoires, etc. - et les comparer aux stratégies énonciatives des discours littéraires et non spécialisés. Son objectif est d'aider l'étudiant à distinguer les caractéristiques de chaque type de discours et de les appliquer aux différentes situations de communication. Pour ce faire, l'enseignant devra disposer de vocabulaires spécialisés à jour, contenant des définitions et des modes d'emploi par niveau de langue. 
Or les vocabulaires sont rarissimes dans les spécialités émergentes. Ceux qui circulent sont tributaires d'une démarche méthodologique qui cherche les articulations notionnelles de ces spécialités davantage dans le texte écrit que dans les productions orales, orales-transcrites ou écrites-oralisées; dans le texte en langue d'origine avant le texte traduit; et qui privilégie le vocabulaire représentatif d'un niveau de langue soutenu, au détriment des vocabulaires de connivence propres aux jargons techniques et aux argots de métier. Les terminologies y sont traitées comme des taxinomies immuables, ce qui occulte le constant renouvellement de la pensée et du langage.

Cette démarche terminologique correspond à la vision classique de la science en tant que dépositaire du savoir véritable, étayé d'un appareil conceptuel monosémique, et du texte scientifique en tant que métalangage inaltérable - vision questionnée par des penseurs aussi différents que Lakatos, Bourdieu, Deleuse et Thuillier et qui convient peu aux spécialités émergentes. Même en IA, un des malentendus qui obligent à reconsidérer tout le problème des systèmes experts est d'avoir créé des modèles déterministes et complets pour des mondes ouverts comme la médecine, qui ne sont jamais clos, mais incomplets, en évolution. On s'y oriente donc vers la création de systèmes experts imparfaits, nuancés, qui se trompent et qui s'améliorent en permanence (Perez 1988:38).

S'il est tout à fait légitime de commencer la recherche terminologique par l'analyse d'un espace rigoureusement délimité et facilement observable de l'intertexte technoscientifique, qui permette d'examiner «in vitro» la génèse d'un concept, sa formulation et sa dénomination par le spécialiste, on devrait pouvoir continuer cette analyse en observant les mêmes phénomènes à l'intérieur d'autres espaces intertextuels et d'en saisir les rapports de manière à «coller» aux variations réelles d'un usage en renouvellement constant.

Dans cette optique méthodologique flexible et ouverte à une connaissance «approchée» du domaine à décrire, les notions IA constituées en diachronie par le jeu du transfert interdisciplinaire, de l'analogie et de la métaphore présentent des écarts variables par rapport aux notions antérieures, écarts observables dans l'apparition ou la disparition de certains traits sémantiques. Ainsi des notions comme «démon», «recuit», «réfraction» ou «agrégat» n'ont pas pris que le pli de l'intelligence artificielle mais aussi, et bien avant, ceux de la physique et des mathématiques. En fait, les notions-clé sont éminemment transférables et ces transferts ne sont pas individuels. Ils entraînent des réseaux notionnels. On comprendra dans ces conditions que les relations entre notions tiennent ensemble le système notionnel et l'IA autant que le noyau dur des primitives sémantiques.

Dans ces transferts susceptibles de nouvelles déterminations il s'établit une filiation des traits sémantiques. Leur ordre d'énumération n'est pas indifférent, on en retrouve certains à l'origine des emplois analogiques et métaphoriques (ex. l'idée d'activation conditionnelle du «démon» et celle de l'alternance réchauffement-refroidissement du «recuit»). D'autres déterminent le choix des modes de dénomination. Un inventaire de ces modes (Cottez: 1987) mettrait bien en évidence les manières dont l'intelligensIA francophone se représente la réalité non linguistique. Il serait intéressant de comparer un tel inventaire aux «principes de dénomination» de l'ISO et aux mécanismes de «déflagration du souvenir» et de la dénomination observés par le cogniticien chez l'expert (Vogel : 1988).

Les apports pluridisciplinaires et l'évolution des notions «propres» à l'IA instaurent une sorte de flou terminologique où la monosémie n'a plus rien d'une délimitation horscontexte du sens des unités discursives. Elle se manifeste, en réaction à la synonymie et à la polysémie, comme tendance désambiguïsante du discours spécialisé. Ainsi, le terme «interprète» désigne selon le contexte, soit un programme interprétateur d'instructions distinct du «compilateur», soit la partie du «moteur d'inférence» d'un système expert responsable de l'interprétation des règles distinctes du «raisonneur», soit le moteur d'infé- 
rence en entier, ou encore une «machine à comprendre» des textes, des paroles, des images, etc. L'on constate déjà une tendance à remplacer «interprète» par «interpréteur» ( $1^{\mathrm{er}}$ sens) et par «interpréteur de règles» $\left(2^{\mathrm{e}}\right.$ sens $)$, ce qui réserverait «interprète» à machine à comprendre».

«Neuronal» et «neuromimétique» renvoient tous les deux à «neurone». Emprunté à la biologie avec le sens «relatif au neurone organique», «neuronal» acquiert en IA l'acception «relatif au neurone formel (ex. ordinateur neuronal») et le synonyme «neuromimétique». (ex. architecture neuromimétique). Mais «neuromimétique» est propre au discours scientifique tandis que «neuronal» se rencontre dans tous les types de discours mentionnés au début de cet exposé. Il y a quatre ans déjà, Yves Gentilhomme observait des phénomènes similaires dans les sciences dites «exactes».

Étudier les procédés terminogènes et les mécanismes de la motivation symbolique en relation avec la typologie des discours, permet de récupérer des parcelles d'ordre dans un désordre apparent, de saisir la spécificité et la perméabilité des niveaux de langue dans une dynamique lexicale inséparable de la production discursive. Certains procédés terminogènes sont exploités davantage dans le vocabulaire standard, d'autres semblent se cantonner dans les vocabulaires de connivence. Ainsi, la dérivation régressive (ex. apporter - apport) est totalement absente du vocabulaire IA standard, tandis que la dérivation impropre (changement de catégorie grammaticale sans ajout ou suppression de suffixes) s'y limite à l'adjectivation des noms construits avec -iel et -ique (ex. linguisticiel, cerviciel, tutoriel) selon le modèle «logiciel» et «informatique».

Toujours au niveau standard, la dérivation propre privilégie les suffixes -tion, -age, et -eur dans la production des noms déverbaux d'action et d'agent (ex. céphalisation, instanciation, squelettisation, résumage, moyennage, piégeage, formaliseur, quantifieur, schématiseur). Les noms d'adjectivaux en -ité désignant des états, des degrés, des qualités concrètes ou abstraites (ex. connectivité, explicabilité, équipotentialité, généralisabilité, satisfaisabilité) l'emportent en nombre sur les noms abstraits en -itude et en -isme (ex. complétude, connexionnisme, indéterminisme).

Dans la catégorie des noms dénominaux, le suffixe -ien désigne les professions nouvelles (ex. cogniticien, algorithmicien, didacticien, systémicien) et dans celle des verbes dénominaux le suffixe -iser est plus productif que -ifier (ex. arboriser, modulariser, squelettiser, complexifier). Les verbes déverbaux du type «courailler» sont inexistants en IA standard. En anglais on retrouve les suffixes équivalents -ize et -ify (ex. skeletonize, fuzzify) mais l'argot américain dérive ses verbes d'onomatopées autant que de noms ou de verbes (ex. to feep - émettre un bruit électronique feutré, différent du strident «beep»; to slurp - absorber une grande quantité de données avant de les analyser ; to crunch - analyser les données en les "grignotant»; to snarf - s'emparer brusquement, avec ou sans permission; to diddle, to twiddle, to tweak - bidouiller; to zorch - transférer à très grande vitesse).

En IA standard, la dérivation parasynthétique n'exploite que quatre particules antérieures sur les dix-huit admises. Ce sont contre-, non-, sous-, et sur- (ex. contrefactuel, non planaire, sous-arbre, surrelaxation). Par contre, les dérivés savants font appel à une vingtaine de morphèmes liés grecs et latins auto-, hétéro-, homo-, hyper-, inter-, macro-, méta-, micro-, mini-, multi-, quasi-, poly-, semi-. On les retrouve tels quels en anglais standard.

Dans les deux langues, l'argot du métier reprend plusieurs de ces procédés avec une nuance affective humour, dérision. («Slang is used for fun, for human communication rather than technical communication» souligne G.S. Steele dans la préface de son Dictionnaire du piocheur. Voici quelques exemples de l'argot américain parlé à Stanford, Yale, Carnegie Mellon et MIT «pessimal» (le pire possible) suit le modèle de 
«optimal»; les suffixes -ity et -itude produisent «mysteriousity», «dubiosity», «disgustitude» et «hackitude»; le suffice -age donne «mumblage» (bredouillage), «winnage» (réussite) et «lossage» (échec); l'adjectif polysémique «bogus» (bidon, à la mords-moile-doigt, incroyable, idiot, inutile) donne «bogosity» (cavillonnerie), «bogon» (fleur de nave), «bogometer» (détecteur de navets en herbe), «bogotify» (rendre bidon), «to bogue out» (fleurir soudainement navet) et «autobogotiphobia» (peur de se rendre zigomar). L'originalité de ces créations est due, en partie tout au moins, à l' «imprévisibilité» recherchée de l'association base de dérivation / suffixe.

Comparée à la dérivation, la composition par jonction (mots-tandem) et par téléscopage (mots-valise) est moins productive en français IA. On y trouve toutefois quelques mots-tandem (langage-objets, point-selle, procédure alpha-bêta) et plusieurs mots-valise (ex. le nom collectif intelligentsIA, les procédures minimax, négamax et delta-min, neuroinformatique, neurophilosophie, etc.). Or, à l'exception d'intelligentsIA, créé en français, tous ces composés sont pris à l'anglais où la composition semble être plus féconde que la dérivation. Cet accueil favorable ne surprend guère, car le français littéraire et le français argotique exploitent le téléscopage à fond. Pensons au «franglais» d'Étiemble, au «fictionnaire» d'Alain Finkielkraut, à l'«éternullité» de Jules Laforge, ou à la «sexofolie» de Montherlant (Amr Ibrahim: 1984). Pensons aussi aux centaines de téléscopages argotiques chez R. Queneau ou San-Antonio.

Les tendances à la polarisation fonctionnelle des procédés lexicaux par niveau de langue sont tout aussi marquées dans la dynamique diachronique de la lexicalisation et de l'abréviation par ellipse ou par siglaison (S. Pavel: 1987).

La diachronie est tout relative puisqu'elle reste en-deça des trente dernières années, mais les exemples y abondent qui montrent l'alternance de l'expansion et du rétrécissement syntagmatique. Ainsi, le terme «propagation» s'applique en IA aux inférences, aux contraintes et aux marqueurs. La propagation sera ascendante, descendante, vers l'avant, vers l'arrière, etc. L'ajout de déterminants fait gagner en précision ce que l'on perd en maniabilité. Mais à l'usage, les syntagmes rétrécissent, la propagation vers l'arrière devient «propagation régressive» et ensuite «rétro-propagation». En anglais, le terme «scanning» se combine avec «upward», «downward», «relational» abrégé d'abord en «up-scanning», «rel-scanning» et ensuite en «upscan», «downscan» et «relscan»; le syntagme périphrase «langage d'interprétation de microprogrammes» est abrégé successivement en «interpréteur microprogrammé», «micro-interpréteur» et «micro-interprète». Le cycle expansion-rétrécissement recommence avec «micro-interprète Prolog» et «microProlog».

L'argot de l'intelligentsIA américaine récupère des syntagmes du vocabulaire scientifque en retient un seul élément (de préférence savant) et le combine avec d'autres tout aussi savants pour créer des syntagmes incompréhensibles au commun des mortels, ou des locutions parallèles à celles de la langue courante. De l'expression mathématique «epsilon-delta proof» où epsilon désigne une quantité plus petite que delta, l'argotier retient «epsilon», le combine avec «squared» (à la puissance deux) et obtient le syntagme «epsilon-squared» au sens de «très peu» ou si l'on préfère «quart-de-poil» (ex. «I got an epsilon-squared improvement»), ou la locution «to be within epsilon of something» (Il s'en faut d'un quart-de-poil). Un savoir enviable est ici mis au profit du mieux-dire humoristique. L'hermétisme y est somme toute plus grégaire que ségrégatif, car les informaticiens affectionnent déjà ces créations de laboratoire IA.

L'argotier emprunte souvent au vocabulaire savant - un programme tombé en panne est qualifé de «catatonic» (suspendu) ou «demented» - mais aussi à la langue courante - le même programme est aussi qualifié de «brain-damaged», l'homme est une «meat-machine» et l'étude du cerveau est «wet engineering» (remarquons la tendance à 
«personnifier» la machine et à «chosifier» l'homme). Parallèlement, le vocabulaire IA standard emprunte à la langue courante et l'argot du métier. Le pays de l'IA abonde en collines, vallées, plateaux et sommets escaladés par des algorithmes. Le raisonnement prend la forme de forêts inductives dont les arbres de décision ont des branches, des racines et des feuilles. Son ciel est plein d'étoiles et de nuées de neurones, son terrain de plages de pixels. Les relations conceptuelles y agissent en cliques, les traits distinctifs s'organisent en cohortes et forment des ilôts de vraisemblance. Les connaissances sont mises en boîte et les règles s'infligent des hara-kiris, engagent des duels, posent des bombes logiques, se transmettent des virus et grignotent des octets. Les concepts ont des ancêtres, des frères et des successeurs qui en héritent les propriétés et les contraintes. Les architectures des machines IA deviennent pyramidales, hypercubiques, neuronales, et systoliques grâce aux puissantes méga-puces dont les plus intelligentes s'avèrent être des schizo-puces. Des procédures domestiques et des diligents ramasse-miettes nettoient leur mémoire pendant que 'des réseaux d'acteurs s'envoient des messages en admirant des affectations sylvestres et des cascades de déduction à travers les lucarnes d'inférence.

Le terminologue peut saisir ces transferts conceptuels et décrire ces véritables navettes d'emprunt entre les niveaux linguistiques en dépouillant une documentation récente, représentative de l'intertexte technoscientifique dans sa globalité, non pas de certaines tranches à l'exclusion d'autres. Le repérage de dénominations d'objets, d'actions et d'attributs sera considérablement simplifié par les techniques du dépouillement automatique.

L'enregistrement et l'interprétation des données terminologiques sont pragmatiques, flexibles et avancent par améliorations successives. Les fiches linguistiques (C. Léonhard: 1988) sont divisées en plusieurs sections, la partie sémantique met en parallèle plusieurs définitions de la notion étudiée et ses relations avec d'autres notions; la partie lexicale indique les termes, leurs synonymes, antonymes et leur contexte d'emploi en combinaisons collocatives. Dans chacune des sections, on peut insérer des observations d'ordre historique (origine, évolution des notions et des termes) ou socio-linguistique (préférences langagières) et des conseils pratiques à l'utilisateur (pouquoi éviter, ne pas confondre, voir aussi, etc.).

S'il travaille sur des fichiers informatisés, le terminologue verse ses fiches, même incomplètes, en fichiers provisoires (les volets $\mathbf{B}$ et $\mathrm{C}$ de la banque Termium, par exemple) quitte à les actualiser progressivement. Mais déjà, il peut amorcer le dialogue avec plusieurs spécialistes du domaine (lettres, questionnaires, rencontres, examen de fiches) afin de combler des lacunes et de trouver des consensus. Ces contacts l'aideront aussi à identifier les problèmes de définition et de dénomination récalcitrants, ceux que les spécialistes eux-mêmes ont du mal à résoudre. En même temps, il notera la réaction des spécialistes devant les créations lexicales qu'ils ne connaissaient peut-être pas.

Le traitement du néologisme en tant que concept essentiellement pragmatique ne peut se limiter à signaler la date de son apparition dans un ouvrage, ou la nouveauté d'une forme par rapport aux formes attestées dans un corpus d'exclusion. Ces repères sont aussi relatifs qu'insuffisants dans une spécialité née il y a trente ans et où les dictionnaires sont pratiquement inexistants. Un adjectif comme «expérientiel» - au sens de «reposant sur l'expérience sans en impliquer nécessairement le recours», sens distinct à la fois d'《empirique» et d'«expérimental» — risque d'être étiqueté d'anglicisme récent si l'on ne prend pas la peine de tracer son histoire dans un domaine comme la philosophie où il fut créé par G. Clemenceau et officiellement adopté par la Société frannçaise de philosophie en 1905.

La ressemblance formelle n'est pas toujours une preuve suffisante de calque ou d'anglicisme: «par défaut» et «sens commun» existent bel et bien en français indépen- 
damment des expressions «default reasoning» et «commonsense rule» très usités en IA. L'expression figurée «idiot savant» qualifie un programme dit «expert» parce que capable d'exécuter des opérations compliquées mais qui s'avère incapable d'un raisonnement heuristique élémentaire. Elle risque d'être prise soit pour un emprunt, soit pour une traduction fautive de l'expression «savant idiot». Or, dans ce cas précis, il s'agit d'un emprunt anglais à la terminologie française de la psychologie génétique où «idiot savant» désigne un handicapé mental qui fait preuve de brillance dans un domaine limité (notons la symétrie avec «âne savant») et non pas un savant abruti. Et que dire du syntagme IA «règle de possibilisation», traduisant «possibilitation rule», sinon que c'est un néologisme d'emprunt puisque introuvable dans les dictionnaires français? Pourtant, sa motivation serait toute autre et son acception facilitée si l'on savait qu'il y a deux cents ans, c'est-àdire en 1784, François Domergue jugeait la série «possibiliser — possibilisation» nécessaire, bien-formée et euphonique (F. Dougnac 1982: 22).

Le traitement des terminologies nouvelles doit tenir compte des acpects psychosociologiques du renouvellement linguistique: un néologisme de forme peut passer inaperçu dans certains milieux tant il reste conforme aux paradigmes dérivationnels les mieux exploités; le nouvel emploi figuré d'un mot courant peut relever d'une motivation symbolique si transparente qu'elle dispense de le définir. En revanche, des termes et des syntagmes bien établis dans l'usage linguistique d'une école de pensée IA seront perçus comme néologismes par une autre et perdront beaucoup de temps à s'y implanter. Avec l'assentiment du spécialiste, le terminologue peut proposer, créer des néologismes, mais il sait que ces créations auront à subir l'épreuve de l'usage au même titre que celles des autres.

La dissémination des terminologies nouvelles est ciblée en fonction des besoins exprimés par leurs utilisateurs parmi lesquels on compte des chercheurs, des étudiants, des traducteurs, des organismes d'aménagement linguistique et de normalisation. La valeur des données dépendra bien entendu de la qualité de la documentation, de la contribution des spécialistes et de la fréquence des mises à jour, mais elle dépendra aussi des connaissances lexicographiques du terminologue attelé à la tâche de décrire une matière pour ainsi dire en fusion.

À la différence de l'informatique classique, l'intelligence artificielle s'accomode de concepts flous, de faits évolutifs, imprécis, incomplets, et même inexacts dans son traitement des connaissances. Le chercheur qui compile et étudie la terminologie IA ne peut ignorer ces techniques de traitement dans ses propres recherches (la communication de Mme Agnès Kukulska en a d'ailleurs illustré l'intérêt).

Le terminologue se trouve un peu dans la situation du cogniticien qui extrait le savoir du médecin ou du géologue et en établit le vocabulaire notionnel dans la base de connaissances d'un système expert. En dépouillant des monographies françaises telles Génie cognitif par C. Vogel, je ne fus qu'à moitié surprise par la similarité des techniques de collecte et de systématisation des donnés en terminologie et en IA, car de toute éviden$\mathrm{ce}$, le terminologue et le cogniticien étudient et interprètent le savoir spécialisé en tant que médiateurs et interlocuteurs de l'expert humain. Ils ne créent pas des notions, ils les font vivre en les véhiculant. Leurs finalités sont toutefois différentes. Le terminologue systématise les moyens d'exprimer ces notions en fonction de la clientèle des usagers possibles, tandis que le cogniticien atomise les connaissances du spécialiste et les formalise en équations logicielles. La représentation formelle du savoir l'emporte ici sur l'expression linguistique des notions. C'est la différence entre la dissémination du savoirdire et la duplication du savoir-faire.

Il existe toutefois un domaine de l'intelligence artificielle où le dire rejoint le faire. Il s'agit du traitement du langage naturel et plus précisément de la création de linguisti- 
ciels pour la recherche terminologique et la traduction automatique. Lorsque le domaine de spécialité de la machine à traduire ou du système expert en terminologie sera l'intelligence artificielle elle-même, le terminologue et le cogniticien se retrouveront sur un terrain commun. Des langages IA évolués décriront le langage naturel de l'IA et cette spécialité se retournera sur elle-même à l'image du serpent mythique Ouroboro rabattu sur sa queue. La boucle du métalangage IA n'est certes pas bouclée mais en anticipation du moment où elle le sera, on pourrait tenter de rapprocher les méthodes d'extraction de l'expertise en IA et celles de la collecte des données en terminologie.

\section{BIBLIOGRAPHIE}

ANDLER, D. et al. (1987): «Intelligence artificielle, sciences cognitives, nature du cerveau», in Le Débat, $\mathrm{n}^{\circ} 47$, Paris, Gallimard.

ARSAC, J. (1987) : Les machines à penser, Paris, Seuil.

BOISVERT, L. et al. (1985) : La lexicologie québécoise - bilan et perspectives, Québec, Les Presses de l'Université Laval.

BOURDIEU, P. (1982): Ce que parler veut dire - l'économie des échanges linguistiques, Paris, Fayard.

CANDEL, D. (1984) : «Ambiguité d'origine polysémique dans une langue de spécialité», in Cahiers de lexicologie, vol. $45, \mathrm{n}^{\circ} 2$.

COTTEZ, H. (1987) : «Sur quelques problèmes de dénomination» in Cahiers de lexicologie, vol. $50, \mathrm{n}^{\circ} 1$.

DELEUZE, G. (1988) : Le pli (Leibniz et le Baroque), Paris, Minuit.

DOUGNAC, F. (1982) : «Aspects de la néologie lexicale», dans le

Journal de la langue françoise (1784-1795), in Linx, Bulletin du Centre de recherches linguistiques de Paris Nanterre, vol. $2, \mathbf{n}^{\circ} 7$.

GENTILHOMME, Y. (1984): «Les faces cachées du discours scientifique - réponse à Jean Peytard» in Langue française, $\mathrm{n}^{\circ}$ 64, Paris, Larousse.

HAGEGE, Cl. (1987) : Le français et les siècles, Paris, Éditions Odile Jacob.

HOTTOIS, G. (1987) : «Technolangues et technophobie philosophique», in Penser l'informatique, informatiser la pensée, Mélanges offerts à André Robinet, Bruxelles, Éditions de l'Université de Bruxelles.

IBRAHIM, A.H. (1984) : «Néologismes par téléscopage», in Le français dans le monde, vol. $23, \mathrm{n}^{\circ} 182$.

LAKATOS, I. (1978): The Methodology of Scientific Research Programmes — Philosophical Papers, Cambridge, Cambridge University Press.

LAURIAN, A.M. et al. (1983): «Typologie des discours scientifiques» in Études de linguistique appliquée, $n^{\circ} 51$, Paris, Didier.

LEONHARD, Ch. (1988) : «Termium : The Structure of its Linguistic Data», in Ata: Technology as Translation Strategy, Binghampton, State University of New York.

MELCUK, I. et al. (1984): Le dictionnaire explicatif et combinatoire du français contemporain - Recherches lexico-sémantiques I, Montréal, Presses de l'Université de Montréal.

MOLINO, J. (1979): «Métaphores, modèles et analogies dans les sciences», in Langages, $n^{\circ} 54$.

PARENT, R. (1988): «Terminologie et modélisation des connaissances» in Terminogramme, $\mathrm{n}^{\circ} 47-48$, Québec, Office de la langue française.

PAVEL, S. (1987) : «La terminologie de l'avenir, un dialogue Homo sentiens-Machina Sapiens», in $M e t a$, vol. $32 \mathrm{n}^{\circ} 2$, Montréal, Presses de l'Université de Montréal.

PAVEL, S. (1988) : «Siglaison et créativité lexicale en intelligence artificielle», in L'actualité terminologique, vol. $21, n^{\circ} 4$, Ottawa, Secrétariat d'État du Canada.

PÉREZ, J.-Cl. (1988) : De nouvelles voies vers l' intelligence artificielle, Paris, Masson.

PEYTARD, J. et al. (1984) : «Français technique et scientifique : reformulation, enseignement», in Langue francaise, $n^{\circ} 64$, Paris, Larousse.

RASTIER, F. et al. (1987): «Sémantique et intelligence artificielle», in Langages, $n^{\circ} 87$, Paris, Larousse.

REY, A. (1984) : «La néologie : un problème de diffusion et d'acceptation», in Actes du colloque national sur les services linguistiques, Ottawa, Secrétariat d'État du Canada.

STEELE, G.S. et al. (1983) : The Hacker's Dictionary, New York, Harper and Row Publ.

THUILLIER, P. (1988): D'Archimède à Einstein - les faces cachées de l'invention scientifique, Paris, Fayard.

VOGEL, C. (1988) : Génie cognitif, Paris, Masson. 